\title{
On the division of distributions by polynomials
}

\author{
By Lars Hörmander
}

\section{Introduction}

The division problem for distributions is, given a distribution $T$ in an open set $\Omega$ in $R^{v}$ and an infinitely differentiable function $\varphi$ in $\Omega$, to find a distribution $S$ in $\Omega$ so that

$$
T=\varphi S
$$

(Cf. Schwartz [4]: Chap. V, pp. 121-126, Chap. VII, p. 154.) One may then also call $S$ a "partie finie" of $T / \varphi$. When $v=1$ the division is possible for every $T$ if and only if $\varphi$ has only isolated zeros of finite order (Schwartz [4], Chap. V, p. 123). When $v>1$, however, the situation is not equally simple. It is the purpose of this paper to prove that the division by a polynomial (not identically zero) is always possible. This was conjectured by Schwartz [4], t. II, p. 154. As indicated there, this also implies that if $T$ is a tempered distribution one can find a tempered "partie finie" $S$-our proof will be arranged so as to give this result directly. By applying the Fourier transformation it follows that every partial differential equation (for notations cf. Hörmander [1])

$$
P(D) u=f
$$

with constant coefficients has a tempered solution u for every tempered $f$. In particular, the equation has a tempered fundamental solution.

By other means, Malgrange [3] and later Hörmander [1] have proved the existence of non tempered fundamental solutions having certain local regularity properties. Such fundamental solutions were called proper by Hörmander [1], and it was also proved in that paper that there are differential equations with no fundamental solution that is both proper and tempered. This shows that the results of this paper are of a character rather different from the earlier ones of Malgrange and Hörmander, and so are the methods of proof.

Let $S$ be the space of infinitely differentiable functions $f$ in $R^{v}$ such that

$$
\sup _{\xi}\left|\xi_{\alpha} D^{\beta} f(\xi)\right|<\infty
$$

for all $\alpha$ and $\beta$. $S$ is a locally convex topological vector space with the topology defined by the semi-norms that are finite according to (1.3) (cf. Schwartz [4]). Our main result is the following. 
L. HöRMANDER, On the division of distributions by polynomials

Theorem 1. If $P$ is a polynomial that does not vanish identically, the multiplication mapping

has a continuous inverse.

$$
S \ni f \rightarrow P f \in S
$$

It may be remarked that the proof remains valid if, instead of being a polynomial, $P$ is any function such that (4.2) and (4.10) are valid.

The essential point in the proof is an application of Whitney's extension theorem for differentiable functions. Since we need certain uniform estimates of the extended functions, which are contained but not explicitly stated in Whitney [6], we shall recall what is needed here in section 3 . Thus the present paper is self-contained in this respect.

While preparing the manuscript, the author has noticed the fact that Lojasiewicz [2] has recently announced a solution of the division problem even for analytic functions instead of polynomials. However, the publication of this paper may still be justified by the differences which seem to exist between his methods and ours. In particular, it seems as though Lojasiewicz' method requires a much more detailed and complicated study of the set of zeros of $P$ than our method does.

\section{Algebraic lemmas}

If $A$ is a set in $R^{v}$, we denote by $d(\xi, A)$ the distance from $\xi$ to $A$,

$$
d(\xi, A)=\inf _{\eta \in A}|\xi-\eta|
$$

Obviously $\left|d(\xi, A)-d\left(\xi^{\prime}, A\right)\right| \leqslant\left|\xi-\xi^{\prime}\right|$, hence $d(\xi, A)$ is a continuous function of $\xi$.

Let $Q$ be a polynomial and $N$ the set of real zeros of $Q$. Assume that $N$ contains some point with $|\xi| \leqslant 1$. Then we have

Lemma 1. There are positive constants $c$ and $\mu$ such that

$$
|Q(\xi)| \geqslant c d(\xi, N)^{\mu},|\xi| \leqslant 1 .
$$

Proof. The lemma follows from a result of Seidenberg and Tarski (cf. Seidenberg [5]). The shortest proof would be to use the results indicated after Theorem 3 in that paper, but since they are not proved in detail we prefer to use only Theorem 3 . (Note that the restriction in the quoted thorem that the coefficients in all polynomials involved shall be rational was removed on p. 372.)

We first look for the conditions on $\xi$ in order that

$$
d(\xi, N)<\delta,
$$

where $\delta$ is a positive number. (2.2) means that there exists a real $\eta$ so that the following equation and inequality hold

$$
Q(\eta)=0,|\xi-\eta|^{2}<\delta^{2}
$$

According to Theorem 3 of Seidenberg [5] one can find a finite number of sets $G_{1}(\xi, \delta)$, $\ldots, \boldsymbol{G}_{r}(\xi, \delta)$, each composed by a finite number of polynomial equations and inequalities in $\xi$ and $\delta$, so that $\left(2.2^{\prime}\right)$ can be fulfilled if and only if $(\xi, \delta)$ satisfies $\boldsymbol{G}_{i}(\xi, \delta)$ for 
at least one $i=1, \ldots, r$. Let $\boldsymbol{H}_{1}(\xi, \delta), \ldots, \boldsymbol{H}_{s}(\xi, \delta)$ be the sets composed by the negation of one condition in each $\boldsymbol{G}_{i}(\xi, \delta)$. Then the negation of (2.2),

$$
d(\xi, N) \geqslant \delta,
$$

is true if and only if $\boldsymbol{H}_{j}$ is fulfilled for at least one $j=1, \ldots, s$.

Next we consider the range of values of $|Q(\xi)|$ when $d(\xi, N) \geqslant \delta,|\xi| \leqslant 1$. Clearly $|Q(\xi)|=\tau$ for some such $\xi$ if and only if there is a real $\xi$ so that

$$
\tau^{2}=|Q(\xi)|^{2}, \tau>0,|\xi|^{2} \leqslant 1, H_{j}(\xi, \delta) \quad \text { holds for some } j=1, \ldots, s .
$$

Again, we have a system of real equations and inequalities to which Seidenberg's theorem applies. Thus we get a finite number of sets $\boldsymbol{K}_{l}(\tau, \delta), l=1, \ldots, t$, of polynomial equations and inequalities so that (2.4) can be satisfied if and only if $\boldsymbol{K}_{l}(\tau, \delta)$ is fulfilled for at least one $l$.

Let $T(\delta)$ be the infimum value of $\tau$ when $\delta$ is fixed, that is, $T(\delta)$ is the infimum of $|Q(\xi)|$ in the compact set $|\xi| \leqslant 1, d(\xi, V) \geqslant \delta$, which is not empty for small $\delta$. Hence the infimum is attained and $>0$. When $\tau=T(\delta)$ some system $K_{l}(\tau, \delta)$ must be satisfied and cannot involve only strict inequalities since all $\tau$ near $T(\delta)$ would then also satisfy $\boldsymbol{K}_{l}(\tau, \delta)$, and $T(\delta)$ would not be the infimum value. Hence $T(\delta)$ always satisfies some of a finite number of algebraic equations in $T$ and $\delta$, and since $T(\delta)$ is decreasing it must therefore be piecewise algebraic because two different algebraic curves have only a finite number of interesections. In particular, $T(\delta)$ is algebraic for small $\delta$. Hence it has a Puiseux expansion for small $\delta$, and we get

$$
T(\delta) \geqslant c_{1} \delta^{\mu}, \quad 0<\delta \leqslant \delta_{0} .
$$

Hence (2.1) holds with $c=c_{1}$ when $0<d(\xi, N) \leqslant \delta_{0}$. It is trivially valid when $d(\xi, \mathrm{N})=$ 0 , and since $T(\delta) \geqslant T\left(\delta_{0}\right)$ when $\delta>\delta_{0}$ we have only to take $c$ somewhat smaller than $c_{1}$ to make (2.1) valid without any additional restrictions on $\xi$. The proof is complete.

In proving Theorem 1 it is convenient-though not necessary-to have an extension of Lemma 1 where the assumption $|\xi| \leqslant 1$ has been dropped.

Lemma 2. Let $Q$ be a polynomial and $N$ the set of its real zeros. Then either $N$ is empty and

$$
|Q(\xi)| \geqslant c\left(1+|\xi|^{2}\right)^{-\mu^{\prime}}, \quad \xi \text { real, }
$$

or else

$$
|Q(\xi)| \geqslant c\left(1+|\xi|^{2}\right)^{-\mu^{\prime}} d(\xi, N)^{\mu^{\prime \prime}}, \quad \xi \text { real, }
$$

where $c>0, \mu^{\prime}$ and $\mu^{\prime \prime}$ are constants.

Proof. It only remains to study the case $|\xi|>1$. To do so we make an inversion

$$
\xi^{*}=\xi /|\xi|^{2}, \quad \xi=\xi^{*} /\left|\xi^{*}\right|^{2}
$$

and write, if $m$ is the degree of $Q$,

$$
Q^{*}\left(\xi^{*}\right)=\left|\xi^{*}\right|^{2 m} Q(\xi)=\left|\xi^{*}\right|^{2 m} Q\left(\xi^{*} /\left|\xi^{*}\right|^{2}\right) .
$$

It is obvious that $Q^{*}$ is a polynomial. Let $N^{*}$ be the set defined by

$$
Q^{*}\left(\xi^{*}\right)=0 \text {. }
$$


L. HörmaNDER, On the division of distributions by polynomials

It is clear that a vector $\xi^{*} \neq 0$ is in $N^{*}$ if and only if the corresponding $\xi$ is in $N$. Applying Lemma 1 to $Q^{*}$ we obtain

$$
\left|Q^{*}\left(\xi^{*}\right)\right| \geqslant c^{*} d\left(\xi^{*}, N^{*}\right)^{\mu *}, \quad \xi^{*} \in N^{*}, \quad\left|\xi^{*}\right| \leqslant 1
$$

where $c^{*}$ and $\mu^{*}$ are positive constants. This means that

$$
|\xi|^{-2 m}|Q(\xi)| \geqslant c^{*} d\left(\xi^{*}, N^{*}\right)^{\mu *}, \quad|\xi| \geqslant 1
$$

Now note that for the inversion we have

$$
|\eta-\xi| /\left|\eta^{*}-\xi^{*}\right|=|\eta||\xi|
$$

hence

$$
d\left(\xi^{*}, N^{*}\right)=\inf _{\eta^{*} \in N^{*}}\left|\eta^{*}-\xi^{*}\right|=\inf _{\eta \in N}|\eta-\xi| /|\eta||\xi|
$$

where we shall permit $\eta$ to be infinite when taking the infimum. We have

$$
|\eta-\xi| /|\eta||\xi| \geqslant d(\xi, N) / 2|\xi|^{2} \text { if }|\eta| \leqslant 2|\xi| \text { and } \eta \in N
$$

and

$$
|\eta-\xi| /|\eta||\xi| \geqslant 1 / 2|\xi| \text { if }|\eta| \geqslant 2|\xi| \text {. }
$$

Since for $|\xi| \geqslant 1$ we always have $d(\xi, N) \leqslant C|\xi|$ for some constant $C$, if $N$ is not empty, it follows that

$$
d(\xi, N) \leqslant C_{1}|\xi|^{2} d\left(\xi^{*}, N^{*}\right), \quad|\xi| \geqslant 1
$$

and (2.7) therefore implies (2.6). On the other hand, if $N$ is empty the only point in $N^{*}$ is 0 , hence $d\left(\xi^{*}, N^{*}\right)=\left|\xi^{*}\right|=1 /|\xi|$ and we obtain (2.5).

Remark. Lojasiewicz [2] states that Lemma 1 still holds if $Q$ is only analytic in the closed unit sphere. If one admits this result, the rest of our arguments still applies with obvious modifications to proving that division by any analytic function is possible.

\section{Whitney's extension theorem}

Whitney [6] has given necessary and sufficient conditions on the array of functions $f^{\alpha},|\alpha| \leqslant m$, defined in a closed set $A$ in $R^{v}$, for the existence of a function $g \in C^{m}\left(R^{v}\right)$ such that $D^{\alpha} g=f^{\alpha}$ in $A$ when $|\alpha| \leqslant m$. (Here $\alpha$ stands for a sequence of indices $\left(\alpha_{1}\right.$, $\ldots, \alpha_{j}$ ) between $I$ and $\nu$, the dimension of the space of $\xi ;|\alpha|=j ; D^{\alpha}=\partial / \partial \xi_{\alpha_{1}} \ldots \partial / \partial \xi_{\alpha_{j}}$.) We shall here use his construction of the extension as an approximation method. Thus we shall, given a function $f \in C^{m}\left(R^{\nu}\right)$, find another function $g \in C^{m}\left(R^{v}\right)$ satisfying

$$
D^{\alpha} g=D^{\alpha} f \text { in } A, \quad|\alpha| \leqslant m,
$$

so that, which is the important point, it is possible to estimate the derivatives of $g$ of order $\leqslant m$ in the whole of $R^{v}$ in terms of quantities involving only those of $f$ of order $\leqslant m$ in $A$.

For the convenience of the reader we shall reproduce the part of Whitney's argument which is needed. No new idea is added, but we modify his argument slightly so that it suits our purposes.

The following fundamental lemma is contained in Whitney [6], pp. 67-69. 
Lemma 3. Given a closed set $A$ in $R^{\nu}$, one can find a sequence of functions $\phi_{j} \in C_{0}^{\infty}(C A)$ with the following properties:

$$
\phi_{j}(\xi) \geqslant 0 ; \quad \sum_{j} \phi_{j}(\xi)=1, \quad \xi \notin A .
$$

(ii) A compact set in $\mathbf{C} A$ intersects only a finite number of the supports of the functions $\phi_{j}$.

$$
\sum_{j}\left|D^{\alpha} \phi_{j}(\xi)\right| \leqslant C_{\alpha}\left(d(\xi, A)^{-|\alpha|}+1\right)
$$

where $C_{\alpha}$ is a constant.

(iv) There is a constant $C$-independent of $j$ and even of $A$-such that the diameter of the support of $\phi_{j}$ is $\leqslant C$ times its distance to $A$.

Proof. Divide $R^{v}$ into a net of cubes of side 1 . Let $K_{0}$ be the set of those cubes with distance to $A$ at least $\sqrt{v}$, the length of the diagonal. Divide the remaining cubes into $2^{v}$ cubes of side $1 / 2$ and let $K_{1}$ be the set of those with distance to $A$ at least equal to the diagonal. With repeated subdivisions we get in this way sets of cubes $K_{0}, K_{1}, \ldots$; the union of all cubes is $\mathrm{C} A$. If a cube $C$ in $K_{i}$ and another $C^{\prime}$ in $K_{9}$, have a point in common, we have $|i-j| \leqslant 1$. For the distance from $C$ to $A$ is $\geqslant 2^{-i} \sqrt{\nu}$, hence a cube with side $2^{-i-1}$ having some point in common with $C$ has distance at least $\sqrt{\nu} 2^{-i-1}$ to $A$, which proves that $j \leqslant i+1$. In view of the symmetry we also have $i \leqslant j+1$, which proves the assertion.

We order all cubes in a sequence $C_{1}, C_{2}, \ldots ;$ the centres are denoted $\eta^{1}, \eta^{2}, \ldots$ and the sides $s_{1}, s_{2} \ldots$ Let $0 \leqslant \phi(\xi) \leqslant 1$ be a function in $C^{\infty}$ which vanishes outside a compact subset of the cube

$$
\left|\xi_{i}\right|<1 / 2+1 / 8, \quad i=1, \ldots, v,
$$

and equals 1 in the smaller cube

$$
\left|\xi_{i}\right| \leqslant 1 / 2, \quad i=1, \ldots, \nu .
$$

We shall prove that the functions

$$
\phi_{j}(\xi)=\phi\left(\left(\xi-\eta^{\xi}\right) / s_{j}\right) / \sum_{\bar{k}} \phi\left(\left(\xi-\eta^{k}\right) / s_{k}\right)
$$

have the properties stated in the lemma.

First note that $\phi\left(\left(\xi-\eta^{k}\right) / s_{k}\right)$ is 0 except in $C_{k}$ and the incident cubes, because these have at least the side length $s_{k} / 2$. Hence

$$
1 \leqslant \sum_{k} \phi\left(\left(\xi-\eta^{k}\right) / s_{k}\right) \leqslant 4^{y}, \quad \xi \notin A,
$$

since at each point at most $4^{v}$ terms are $\neq 0$ and at least one equals 1 . This proves that $\phi_{j} \in C_{0}^{\infty}(\mathrm{C} A)$ and that (i) and (ii) are valid is obvious. To prove (iv) we note that $\phi_{i}=0$ outside the cube with side $s_{i}(1+1 / 4)$ with centre at $\eta^{i}$; the distance from that cube to $A$ is at least

$$
\sqrt{\nu} s_{i}-\sqrt{v} s_{i} / 8=7 \sqrt{v} s_{i} / 8
$$

and its diameter is $5 \sqrt{\nu} s_{i} / 4$. Hence (iv) holds with $C=10 / 7$. 


\section{HöRMANDER, On the division of distributions by polynomials}

Finally, in proving (iii) we note that, with $\phi^{(\alpha)}=D^{\alpha} \phi$,

$$
D^{\alpha}\left(\phi\left(\left(\xi-\eta^{j}\right) / s_{j}\right)\right)=s_{j}^{-|\alpha|} \phi^{(\alpha)}\left(\left(\xi-\eta^{j}\right) / s_{j}\right) .
$$

The distance from the support of $\phi^{(\alpha)}\left(\left(\xi-\eta_{j}\right) / s_{j}\right)$ to $A$ is at most $3 s_{j} \sqrt{v}$ if $s_{j}<1$. For otherwise the cube with side $2 s_{j}$ containing $C$, in the preceding decomposition would have distance at least $2 s_{j} \sqrt{\nu}$ from $A$, which contradicts the construction. Hence

$$
d(\xi, A)<5 s_{j} \sqrt{\nu} \text { if } s_{j}<1 \text { and } \phi^{(\alpha)}\left(\left(\xi-\eta^{j}\right) / s_{j}\right) \neq 0 .
$$

If $s_{j}=1$ we have on the other hand

$$
d(\xi, A) \geqslant 7 \sqrt{v} / 8>1 \text { if } \phi^{(\alpha)}\left(\left(\xi-\eta^{j}\right) / s_{j}\right) \neq 0 .
$$

Summing up, we obtain if $d(\xi, A) \leqslant 1$

$$
\left|D^{\alpha} \phi\left(\left(\xi-\eta^{j}\right) / s_{j}\right)\right| \leqslant\left(\sup \left|\phi^{(\alpha)}\right|\right)(5 \sqrt{\nu})^{|\alpha|} d(\xi, A)^{-|\alpha|}, \quad j=1,2, \ldots
$$

and if $d(\xi, A) \geqslant 1$ we get, since the only non vanishing functions have $s_{j}>1 / 5 \sqrt{\nu}$,

$$
\left|D^{\alpha} \phi\left(\left(\xi-\eta^{j}\right) / s_{j}\right)\right| \leqslant\left(\sup \left|\phi^{(\alpha)}\right|\right)(5 \sqrt{\nu})^{|\alpha|}, \quad j=1,2, \ldots
$$

Using these two estimates and (3.3) in (3.2), taking into account that no point $\xi$ is in the support of more than $4^{v}$ terms $\phi\left(\left(\xi-\eta^{k}\right) / s_{k}\right)$, we obtain the inequality (iii).

For every $j$ we choose a point $\xi^{i} \in A$ such that the distance from the support of $\phi$, to $A$ equals the distance to $\xi^{\dagger}$. Given a function $f \in C^{m}\left(R^{v}\right)$ we now set (Whitney $[6$, p. 69])

$$
g(\xi)=\Sigma^{*} \phi_{j}(\xi) f_{m}\left(\xi ; \xi^{\xi}\right), \quad \xi \notin A ; \quad g(\xi)=f(\xi), \xi \in A
$$

where

$$
f_{m}(\xi ; \eta)=\sum_{|\alpha| \leqslant m} D^{\alpha} f(\eta)(\xi-\eta)_{\alpha} /|\alpha| !
$$

is the Taylor expansion for $f$ of order $m$ at $\eta$. We are going to prove that (3.1) is valid for the function $g$ defined by (3.4) and then estimate $g$ and its derivatives. The notation $\Sigma^{*}$ in (3.4) means that we only sum over those $j$ with $s_{j}<1$. This changes nothing near $A$ but may improve the behaviour of $g$ at infinity; we get $g(\xi)=0$ if $d(\xi, \mathrm{A})>5 \sqrt{\nu} / 2$.

Before studying $g$ we introduce some more notations. By $R_{m}(\xi ; \eta)$ we denote the remainder in Taylor's formula,

$$
f(\xi)=f_{m}(\xi ; \eta)+R_{m}(\xi ; \eta)
$$

with the notation $F^{(\alpha)}=D^{\alpha} F$ we obtain by differentiating

$$
f^{(\alpha)}(\xi)=f_{m}^{(\alpha)}(\xi ; \eta)+R^{(\alpha)}(\xi ; \eta)
$$

where the differentiations act on the first variable in $f_{m}$ and in $R_{m}$.

Let $\xi^{*} \in A$ be a point with minimal distance to $\xi$,

$$
\left|\xi^{*}-\xi\right|=d(\xi, A), \quad \xi \notin A .
$$


We shall compare $g^{(\alpha)}(\xi)$ with $f_{m}^{(\alpha)}\left(\xi ; \xi^{*}\right)$. Assuming that $d(\xi, A)<1$ we have $\Sigma^{*} \phi_{j}(\xi)=1$ and hence

$$
g(\xi)=f_{m}\left(\xi ; \xi^{*}\right)+\sum_{j}^{*} \phi_{j}(\xi)\left(f_{m}\left(\xi ; \xi^{\xi}\right)-f_{m}\left(\xi ; \xi^{*}\right)\right) .
$$

Differentiating we get using Leibniz' formula

$$
g^{(\alpha)}(\xi)=f_{m}^{(\alpha)}\left(\xi ; \xi^{*}\right)+\sum_{\beta+\gamma=\alpha} \sum_{j}^{*} \phi_{j}^{(\beta)}(\xi)\left(f_{m}^{(\gamma)}\left(\xi ; \xi^{\xi}\right)-f_{m}^{(\gamma)}\left(\xi ; \xi^{*}\right)\right) .
$$

If $\xi$ is in the support of $\phi_{j}$, the distance from this support to $A$ is at most $\left|\xi-\xi^{*}\right|$, hence the diameter is $\leqslant C\left|\xi-\xi^{*}\right|$ in virtue of (iv) and, in view of the definition of $\xi^{i}$,

$$
\left|\xi-\xi^{j}\right| \leqslant(C+1)\left|\xi-\xi^{*}\right|=(C+1) d(\xi, A) \text {. }
$$

Hence also

$$
\left|\xi^{j}-\xi^{*}\right| \leqslant(C+2)\left|\xi-\xi^{*}\right|=(C+2) d(\xi, A) \text {. }
$$

Introducing remainder terms in (3.7) we obtain

$$
g^{(\alpha)}(\xi)=f^{(\alpha)}(\xi)-R_{m}^{(\alpha)}\left(\xi ; \xi^{*}\right)+\sum_{\beta+\gamma=\alpha} \sum_{j}^{*} \phi_{j}^{(\beta)}(\xi)\left(R_{m}^{(\gamma)}\left(\xi ; \xi^{j}\right)-R_{m}^{(\gamma)}\left(\xi ; \xi^{*}\right)\right) .
$$

When $\xi$ and $\eta$ belong to a fixed compact set, the classical expressions for the remainder term give

$$
\left|R_{m}^{(\gamma)}(\xi ; \eta)\right| \leqslant|\xi-\eta|^{m-|\gamma|} \varepsilon(|\xi-\eta|)
$$

where $\varepsilon(t)$ decreases to 0 with $t$. With $d=\left|\xi-\xi^{*}\right|=d(\xi, A)$ and $d_{1}==(C+1) d$, we get using condition (iii) of Lemma 3 and (3.8)

$$
\left|g^{(\alpha)}(\xi)-f^{(\alpha)}(\xi)\right| \leqslant d^{m-|\alpha|} \varepsilon(d)+\sum_{\beta+\gamma=\alpha} 2 C_{\beta} d^{-|\beta|}\left(d_{1}^{m-|\gamma|} \varepsilon\left(d_{1}\right)+d^{m-|\gamma|} \varepsilon(d)\right) \rightarrow 0
$$

when $d \rightarrow 0$ since $m-|\beta|-|\gamma|=m-|\alpha| \geqslant 0$. Let $h=f-g$. Then $h=0$ in $A$ and $h \in C^{m}(C A)$, the derivatives of order $\leqslant m$ tending to 0 when $\xi \rightarrow A$. An elementary argument which we do not elaborate shows that $h$ is then in $C^{m}\left(R^{v}\right)$ and that its derivatives vanish in $A$. This proves that $g \in C^{m}\left(R^{v}\right)$ and satisfies (3.1).

It remains for us to obtain an estimate for $g^{(\alpha)}$ which only involves the values of $f$ and its derivatives in $A$.

Definition. If $B$ is a set in $R^{v}$ and $f \in C^{m}\left(R^{\nu}\right)$, we denote by $|f|_{m, B}$ the least upper bound of the quantities

$$
\begin{gathered}
\left|f^{(\alpha)}(\xi)\right|, \quad|\alpha| \leqslant m, \quad \xi \in B, \\
\text { and } \quad\left|R_{m}^{(\alpha)}(\xi ; \eta)\right| /|\xi-\eta|^{m-|\alpha|}, \quad|\alpha| \leqslant m, \quad \xi \neq \eta ; \quad \xi, \eta \in B .
\end{gathered}
$$

If $B$ is empty we define $|f|_{m, B}=0$, and if $B$ consists of the point $\xi$ only we write $|f|_{m, \xi}$ instead of $|f|_{m, B}$, thus

$$
|f|_{m, \xi}=\sup _{|\alpha| \leqslant m}\left|f^{(\alpha)}(\xi)\right| \text {. }
$$

Let $A_{\xi}$ be the set of points in $A$ with distance at most $R=(C+1) 5 \sqrt{v} / 2$ from $\xi$. We shall prove that there is a constant $K_{1}$, independent of $f$ and $\xi$, so that for the function $g$ defined by (3.4) we have 
L. HörmaNDER, On the division of distributions by polynomials

$$
|g|_{m, \xi} \leqslant K_{1}|f|_{m, A_{\xi}}, \quad \xi \in R^{v} .
$$

This inequality is obvious if $d(\xi, A) \geqslant 5 \sqrt{\nu} / 2$ for then all terms in (3.4) are 0 . Next note that if $d(\xi, A) \leqslant 5 \sqrt{v} / 2$ we have $\xi^{*} \in A_{\xi}$ and $\xi^{j} \in A_{\xi}$ if $\xi$ is in the support of $\phi_{j}$. This follows from (3.8). have

When $1 \leqslant d(\xi, A) \leqslant 5 \sqrt{\nu} / 2$, the equation (3.7) does not necessarily hold but we

$$
g^{(\alpha)}(\xi)=\sum_{\beta+\gamma=\alpha} \sum_{j}^{*} \phi_{j}^{(\beta)}(\xi) f_{m}^{(p)}\left(\xi ; \xi^{j}\right)
$$

For all $j$ with $\phi_{j}^{(\beta)}(\xi) \neq 0$ we can estimate

$$
f_{m}^{(\gamma)}\left(\xi ; \xi^{\prime}\right)=\sum_{|\beta| \leq m-|\gamma|} f^{(\gamma+\beta)}\left(\xi^{\xi}\right)\left(\xi-\xi^{j}\right)_{\beta} /|\beta| !
$$

by a constant multiple of $|f|_{m, A_{\xi}}$ because $\xi^{j} \in A_{\xi}$ and $\left|\xi-\xi^{j}\right| \leqslant R$. If we then apply property (iii) in Lemma 3, noting that $d(\xi, A) \geqslant 1$, inequality (3.12) follows in this case.

When $0<d(\xi, A)<1$, the only remaining case to study, we can use (3.7). The term $f_{m}^{(\alpha)}\left(\xi, \xi^{*}\right)$ can obviously be estimated by a multiple of $|f|_{m, A_{\xi}}$. What we need is thus only an estimate of the difference

$$
f_{m}^{(\gamma)}\left(\xi ; \xi^{\xi}\right)-f_{m}^{(\gamma)}\left(\xi ; \xi^{*}\right)
$$

when $\xi^{j}$ and $\xi^{*} \in A_{\xi}$. We have

Hence

$$
\begin{aligned}
f_{m}^{(\gamma)}\left(\xi ; \xi^{j}\right) & =\sum_{|\beta| \leqslant m-|\gamma|} f^{(\gamma+\beta)}\left(\xi^{j}\right)\left(\xi-\xi^{j}\right)_{\beta} /|\beta| ! \\
& =\sum_{|\beta| \leqslant m-|\gamma|}\left(f_{m}^{(\gamma+\beta)}\left(\xi^{j} ; \xi^{*}\right)+R_{m}^{(\gamma+\beta)}\left(\xi^{j} ; \xi^{*}\right)\right)\left(\xi-\xi^{j}\right)_{\beta} /|\beta| ! \\
& =f_{m}^{(\gamma)}\left(\xi ; \xi^{*}\right)+\sum_{|\beta| \leqslant m-|\gamma|} R_{m}^{(\gamma+\beta)}\left(\xi^{j} ; \xi^{*}\right)\left(\xi-\xi^{j}\right)_{\beta} /|\beta| !
\end{aligned}
$$

$$
\begin{aligned}
& \left|f_{m}^{(\gamma)}\left(\xi ; \xi^{j}\right)-f_{m}^{(\gamma)}\left(\xi ; \xi^{*}\right)\right|=\left|\sum R_{m}^{(\gamma+\beta)}\left(\xi^{j} ; \xi^{*}\right)\left(\xi-\xi^{j}\right)_{\beta} /\right| \beta|!| \\
& \quad \leqslant|f|_{m, A_{\xi}} \sum\left|\xi^{\xi}-\xi^{*}\right|^{m-|\gamma|-|\beta|}\left|\xi-\xi^{j}\right|^{|\beta|} /|\beta| ! \leqslant K_{1}|f|_{m, A_{\xi}} d(\xi, A)^{m-|\gamma|}
\end{aligned}
$$

in virtue of (3.8) and (3.9); $K_{1}$ is a constant. But using this estimate and condition (iii) of Lemma 3 in (3.7) we get the desired result since $d(\xi, A)$ will occur in the power $m-|\gamma|-|\beta|=m-|\alpha| \geqslant 0$.

We collect as a theorem the results of Whitney given in this section.

Theorem 2. The linear operator $f \rightarrow g$ defined by (3.4) maps $C^{m}\left(R^{v}\right)$ into itself -so that (3.1) and (3.12) are valid.

\section{Proof of Theorem 1}

Let $P$ be a polynomial $\neq 0$. Since Theorem 1 is trivial if $P$ is a constant, we assume that it is of positive degree $\mu$. By $N^{k}$ we denote the set of all real $\xi$ where $P$ has a zero of order $k$ at least. Thus $N^{k}$ is the closed set defined by the equations 


$$
P^{(\alpha)}(\xi)=0, \quad|\alpha|<k, \quad \xi \in R^{\nu} .
$$

Since $P^{(\alpha)}=$ constant $\neq 0$ for some $\alpha$ with $|\alpha|=\mu$, we have $N^{\mu+1}=\phi$, thus

$$
R^{v}=N^{0} \supset N^{1} \supset N^{2} \supset \cdots \supset N^{\mu+1}=\phi \text {. }
$$

The idea of the proof of Theorem 1 consists in estimating $f$ in terms of $P f$ first in $N^{\mu}$, then in $N^{\mu-1}$ and so on. Thus we shall prove successively for decreasing $k$ the following statement, which is equivalent to Theorem 1 when $k=0$ and is trivial when $k=\mu+1$.

To all non negative integers $n, m$ and $k \leqslant \mu$ there are non negative integers $n^{\prime}, m^{\prime}$ and a constant $K$ such that

$$
\sup _{\xi}(1+|\xi|)^{n}|f|_{m, N_{\xi}^{k}} \leqslant K \sup _{\xi}(1+|\xi|)^{n^{\prime}}|P f|_{m^{\prime}, \xi}, \quad f \in C^{m^{*}}\left(R^{v}\right)
$$

In proving this result we may of course assume that the corresponding statement with $k$ replaced by $k+1$ is already established.

We start by giving some formulas for the quantities occurring in the definition of $|f|_{m, N_{\xi}^{k}}$ (cf. section 3). With

$$
P(\xi) f(\xi)=F(\xi)
$$

we get in view of (4.1) by applying to (4.4) the differential operator $D^{\beta},|\beta|=k$, that

$$
P^{(\beta)}(\xi) f(\xi)=F^{x(\beta)}(\xi), \quad \xi \in N^{k}, \quad|\beta|=k
$$

In order to obtain a similar formula for $f^{(\alpha)}(\xi)$ we write

$$
f(\xi)=F(\xi) / P(\xi)
$$

when $P(\xi) \neq 0$, apply the differential operator $D^{\alpha}$ to both sides and multiply by $P(\xi)^{|\alpha|+1}$ afterwards. This gives, for all $\xi \in R^{\nu}$,

$$
P(\xi)^{|\alpha|+1} f^{(\alpha)}(\xi)=L_{|\alpha|}(\xi, F),
$$

if we use the convention that $L_{s}$ denotes a differential operator acting on $F$ of order $s$ with coefficients that are polynomials in $\xi$. Applying the differential operator $D^{\beta}$, $|\beta|=(|\alpha|+1) k$, we get

$$
\left\{D^{\beta}\left(P(\xi)^{|\alpha|+1}\right)\right\} f^{(\alpha)}(\xi)=L_{k|\alpha|+k+|\alpha|}(\xi, F), \quad \xi \in N^{k}, \quad|\beta|=(|\alpha|+1) k .
$$

We also need a formula for the Taylor remainder terms $R_{m}^{(\alpha)}(\xi ; \eta)$ with $\xi, \eta \in N^{k}$. By definition we have

$$
R_{m}^{(\alpha)}(\xi ; \eta)=f^{(\alpha)}(\xi)-\sum_{|\beta| \leqslant m-|\alpha|} f^{(\alpha+\beta)}(\eta)(\xi-\eta)_{\beta} /|\beta| !
$$

Multiplying by $P(\xi)^{|\alpha|+1} P(\eta)^{m+1}$ we get using (4.6)

$$
\begin{aligned}
& P(\xi)^{|\alpha|+1} P(\eta)^{m+1} R_{m}^{(\alpha)}(\xi ; \eta) \\
& \quad=P(\eta)^{m+1} L_{|\alpha|}(\xi, F)-P(\xi)^{|\alpha|+1} \sum_{|\beta| \leqslant m-|\alpha|} P(\eta)^{m-|\alpha|-|\beta|} L_{|\alpha|+|\beta|}(\eta, F) \frac{(\xi-\eta)_{\beta}}{|\beta| !} \\
& \quad=L_{|\alpha|, m}(\xi, \eta, F) .
\end{aligned}
$$




\section{HöRMANDER, On the division of distributions by polynomials}

Here and in what follows we understand by $L_{s, t}(\xi, \eta, F)$ a linear combination of the derivatives of $F$ at $\xi$ and at $\eta$ of order $\leqslant s$ and $\leqslant t$ respectively, with coefficients that are polynomials in $\xi$ and $\eta$. For later purposes we note that all derivatives of $L_{|\alpha|, m}(\xi, \eta, F)=P(\xi)^{|\times|+1} P(\eta)^{m+1} R_{m}^{(\alpha)}(\xi ; \eta)$ with respect to $\xi$ and $\eta$ of total order $<k(|\alpha|+1)+k(m+1)+m-|\alpha|$ vanish if $\xi=\eta \in N^{k}$. In fact, to get a non zero term in the expression for a derivative given by Leibniz' formula one has to differentiate at least $k(|\alpha|+1)$ times on the factor $P(\xi)^{|\alpha|+1}, k(m+1)$ times on the next factor $P(\eta)^{m+1}$ and $m-|\alpha|$ times on the factor $R_{m}^{(\alpha)}(\xi ; \eta)$.

Applying the differential operator $D^{\beta}$ with respect to $\xi$ and $D^{\gamma}$ with respect to $\eta$ in $(4.8)$, we obtain in view of $(4.1)$

$$
\begin{gathered}
\left\{D^{\beta}\left(P(\xi)^{|\alpha|+1}\right)\right\}\left\{D^{\gamma}\left(P(\eta)^{m+1}\right)\right\} R_{m}^{(\alpha)}(\xi ; \eta)=L_{k|\alpha|+k+|\alpha|, k m+k+m}(\xi, \eta, F) ; \\
\xi, \eta \in N^{k}, \quad|\beta|=(|\alpha|+1) k, \quad|\gamma|=(m+1) k .
\end{gathered}
$$

From a remark made above it follows that the function $L_{k|\alpha|+k+|\alpha|, k m+k+m}(\xi, \eta, F)$, which is the right hand side of (4.9), vanishes of order $m-|\alpha|$ if $\eta$ is fixed in $N^{k}$ and $\xi \rightarrow \eta$ (without necessarily belonging to $N^{k}$ ). This will be important in establishing a favourable estimate of $R_{m}^{(\alpha)}$ by means of Taylor's formula.

We are now going to prove that $m^{\prime}$ and $n^{\prime}$ can be found so that (4.3) is valid under the additional assumption that $f$ vanishes of order $m^{\prime}$ in $N^{k+1}$. (If $N^{k+1}=\phi$ this is of course no restriction.) The proof will follow in this case from the formulas (4.7) and (4.9) and application of Lemma 2. Using Whitney's theorem and the hypothesis that a result of the form (4.3) is valid when $k$ is replaced by $k+1$, we will then be able to complete the proof.

We assume that $N^{k+1}$ is not empty-the opposite case is simpler and is covered by the following arguments with obvious modifications. Lemma 2 applied to the polynomial

$$
Q(\xi)=\sum_{|\beta| \leqslant j k}\left|D^{\beta}\left(P(\xi)^{j}\right)\right|^{2}
$$

which vanishes precisely in $N^{k+1}$ then shows that there are positive constants $c$, $\mu^{\prime}, \mu^{\prime \prime}$ such that

$$
\sum_{|\beta|=j k}\left|D^{\beta}\left(P(\xi)^{j}\right)\right|^{2} \geqslant c d\left(\xi, N^{k+1}\right)^{2 \mu^{r}}\left(1+|\xi|^{2}\right)^{-\mu^{\prime r}}, \quad \xi \in N^{k} .
$$

(Note that the derivatives of $P^{j}$ of order $<j k$ all vanish in $N^{k}$.) The constants depend on $j$. (Actually it is easy to prove that one can choose $\mu^{\prime}$ and $\mu^{\prime \prime}$ proportional to $j$ ). We shall denote by $\mu_{m}^{\prime}$ and $\mu_{m}^{\prime \prime}$ two constants so large that (4.10) holds with $\mu^{\prime}=\mu_{m}^{\prime}$, $\mu^{\prime \prime}=\mu_{m}^{\prime \prime}$ and some $c>0$ for all $j \leqslant(m+1)$.

Let $m^{\prime}$ be an integer so large that

$$
m^{\prime} \geqslant \mu_{m}^{\prime}+k|\alpha|+k+|\alpha| \text {. }
$$

We shall prove that for suitable constants $n^{\prime}$ and $K$, independent of $f$ and $\xi$,

$$
\left|f^{(\alpha)}(\xi)\right| \leqslant K(1+|\xi|)^{n^{\prime}}|F|_{m^{\prime}, \mathrm{s}(\xi)}, \quad \xi \in N^{k},
$$

where $S(\xi)$ is the sphere with centre at $\xi$ and fixed radius $=1$, provided that $f$ vanishes of order $m^{\prime}$ in $N^{k+1}$. This will follow from (4.7) and (4.10) when we have proved the following estimate of the right hand side of (4.7): 
ARKIV För MATEMATIK. Bd $3 \mathrm{nx} 53$

$\left|L_{k|\alpha|+k+|\alpha|}(\xi, F)\right| \leqslant K_{1}(1+|\xi|)^{n_{1}} d\left(\xi, N^{k+1}\right)^{m^{\prime}-k|\alpha|-k-|\alpha|}|F|_{m^{\prime}, s(\xi)}, \quad \xi \in R^{v}$

where $K_{1}$ and $n_{1}$ are constants. (4.13) is obvious if $d\left(\xi, N^{k+1}\right) \geqslant 1$ because $m^{\prime} \geqslant$ $k|\alpha|+k+|\alpha|$. If $d\left(\xi, N^{k+1}\right)<1$ there is a point $\xi^{*} \in N^{k+1}$ such that $\left|\xi^{*}-\xi\right|=$ $d\left(\xi, N^{k+1}\right)$, hence $\xi^{*} \in S(\xi)$. At $\xi^{*}$ the function $L_{k|\alpha|+k+|x|}(\xi, F)$ vanishes of order $m^{\prime}-k|\alpha|-k-|\alpha|$ at least since $F=P f$ vanishes of order $m^{\prime}$. Hence by Taylor's theorem applied to the Taylor expansion of the function $L_{k|\alpha|+k+|\alpha|}(\xi, F)$ at $\xi^{*}$

$$
\left|L_{k|\alpha|+k+|\alpha|}(\xi, F)\right| \leqslant\left(\nu\left|\xi-\xi^{*}\right|\right)^{m^{\prime}-k|\alpha|-k-|\alpha|}\left|L_{k|\alpha|+k+|\alpha|}(\eta, F)\right|_{m^{\prime}-k|\alpha|-k-|\alpha|, S(\xi)}
$$

which proves (4.13). Squaring and adding the equations (4.7) with absolute values taken on both sides, we now get in view of (4.13), if $\xi \in N^{k}$,

$$
\begin{aligned}
\left\{\sum_{|\beta|-(|\alpha|+1) k} \mid D^{\beta}(\right. & \left.\left.P(\xi)^{|\alpha|+1}\right)\left.\right|^{2}\right\}^{\frac{1}{2}}\left|f^{(\alpha)}(\xi)\right| \leqslant \\
& \leqslant K_{2}(1+|\xi|)^{n_{2}} d\left(\xi, N^{k+1}\right)^{m^{\prime}-k|\alpha|-k-|\alpha|}|F|_{m^{\prime}, S(\xi)}
\end{aligned}
$$

and applying (4.10) we get

$$
\left|f^{(\alpha)}(\xi)\right| \leqslant K_{3}(\mathrm{I}+|\xi|)^{n_{3}} d\left(\xi, N^{k+1}\right)^{m^{\prime}-\mu_{m}^{\prime}-k|\alpha|-k-|\alpha|}|F|_{m^{\prime}, S(\xi)}, \quad \xi \in N^{k} .
$$

This implies (4.12) in virtue of (4.11).

We shall now establish an estimate of the form (4.12) for the quantities

$$
R_{m}^{(\alpha)}(\xi ; \eta) /|\xi-\eta|^{m-|\alpha|} ; \quad \xi, \eta \in N^{h} .
$$

In doing so we distinguish between two different cases.

CASE I: In this case we assume that the points $\xi$ and $\eta$ lie so far apart that

$(4.12)^{\prime}$ shows that if

$$
2|\xi-\eta| \geqslant d\left(\xi, N^{k+1}\right)+d\left(\eta, N^{k+1}\right) \text {. }
$$

$$
m^{\prime} \geqslant m+\mu_{m}^{\prime}+k|\alpha|+k+|\alpha|
$$

there exist constants $n^{\prime}$ and $K$ such that

$$
\left|f^{(\alpha)}(\xi)\right| \leqslant K(1+|\xi|)^{n^{\prime}} d\left(\xi, N^{k+1}\right)^{m}|P f|_{m^{\prime}, S(\xi)}, \quad \xi \in N^{k},
$$

if $f$ vanishes of order $m^{\prime}$ in $N^{k+1}$. Now $R_{m}^{(\alpha)}(\xi ; \eta)$ is a linear combination of derivatives of $f$ at $\xi$ and at $\eta$ of order $\leqslant m$, multiplied by polynomials in $\xi$ and $\eta$. If (4.14) holds we can use the estimates $d\left(\xi, N^{k+1}\right) \leqslant 2|\xi-\eta|$ and $d\left(\eta, N^{k+1}\right) \leqslant 2|\xi-\eta|$ in $(4.12)^{\prime \prime}$, which holds for all $\alpha$ with $|\alpha| \leqslant m$ provided that

$$
m^{\prime} \geqslant m+\mu_{m}^{\prime}+k m+k+m .
$$

Hence we have with new constants $K$ and $n^{\prime}$ provided that $(4.11)^{\prime \prime}$ and (4.14) are valid:

$$
\left|R_{m}^{(\alpha)}(\xi ; \eta)\right| \leqslant K(1+|\xi|+|\eta|)^{n^{\prime}}|\xi-\eta|^{m}|P f|_{m^{\prime}, S(\xi, \eta)}, \quad \xi, \eta \in N^{k}
$$

if $S(\xi, \eta)=S(\xi) \cup S(\eta)$ and $f$ vanishes of order $m^{\prime}$ in $N^{k+1}$. 
L. HÖRMANDER, On the division of distributions by polynomials

CASE II: In this case our assumption, opposite to (4.14), is that the points $\xi$ and $\eta$ are so close that

$$
2|\xi-\eta|<d\left(\xi, N^{k+1}\right)+d\left(\eta, N^{k+1}\right) .
$$

Since

$$
\left|d\left(\xi, N^{k+1}\right)-d\left(\eta, N^{k+1}\right)\right| \leqslant|\xi-\eta|,
$$

if follows that

$$
d\left(\eta, N^{k+1}\right) / 3<d\left(\xi, N^{k+1}\right)<3 d\left(\eta, N^{k+1}\right) .
$$

If $\zeta$ is a point on the line segment joining $\xi$ and $\eta$ we also have

$$
\left.\begin{array}{rl}
d\left(\zeta, N^{k+1}\right) \leqslant|\zeta-\xi|+d\left(\xi, N^{k+1}\right) & \leqslant|\eta-\xi|+d\left(\xi, N^{k+1}\right)<3 d\left(\xi, N^{k+1}\right), \\
d\left(\zeta, N^{k+1}\right) & <3 d\left(\eta, N^{k+1}\right) .
\end{array}\right\}
$$

We now use (4.9). As pointed out after that formula, the function

$$
H(\zeta)=L_{k|\propto|+k+|\alpha|, k m+k+m}(\zeta, \eta, F),
$$

where $\eta$ is fixed in $N^{k}$, vanishes of order $m-|\alpha|$ for $\zeta=\eta$. If we denote by $\vartheta$ the unit vector $(\xi-\eta) /|\xi-\eta|$ and by $\langle D, \vartheta\rangle$ the differentiation in the direction $\vartheta$ with respect to $\zeta$, Taylor's formula applied to the function $H$ gives

$$
\begin{array}{r}
\mid L_{k|\alpha|+k+|\alpha|, k m+k+m}\left(\xi, \eta, F|\leqslant| \xi-\left.\eta\right|^{m-|\alpha|}\left|\langle D, \vartheta\rangle^{m-|\alpha|} H(\zeta)\right| /(m-|\alpha|) !,\right. \\
\eta \in N^{k},
\end{array}
$$

for some $\zeta$ in the line segment from $\xi$ to $\eta$. Now $\langle D, \vartheta\rangle^{m-|\alpha|} H(\zeta)$ is a combination with uniformly bounded coefficients (products of coordinates for $\vartheta$ ) of polynomials in $\zeta$ and $\eta$ multiplied with derivatives of $F$ at $\zeta$ and at $\eta$ of order not exceeding $k m+k+m$.

If

$$
m^{\prime} \geqslant 2 \mu_{m}^{\prime}+k m+k+m
$$

and $f$ vanishes of order $m^{\prime}$ in $N^{k+1}$, we get by arguing as in the proof of (4.13) since all derivatives of $F$ occurring in the right hand side of (4.19) vanish of order $m^{\prime}-k m-k-m \geqslant 2 \mu_{m}^{\prime}$ in $N^{k+1}$

$$
\begin{array}{r}
\left|D^{\beta}\left(P(\xi)^{|\alpha|+1}\right)\right|\left|D^{\gamma}\left(P(\eta)^{m+1}\right)\right|\left|R_{m}^{(\alpha)}(\xi ; \eta)\right|=\left|L_{k|\alpha|+k+|\alpha|, k m+k+m}(\xi, \eta, F)\right| \\
\leqslant K_{4}|\xi-\eta|^{m-|\alpha|}\left(d\left(\eta, N^{k+1}\right)+d\left(\zeta, N^{k+1}\right)\right)^{2 \mu^{\prime} m}(1+|\eta|+|\zeta|)^{n_{1}}\left|F^{\prime}\right|_{m^{\prime}, s_{1}(\xi, \eta)} \\
|\beta|=(|\alpha|+1) k, \quad|\gamma|=(m+1) k, \quad \xi, \eta \in N^{k}
\end{array}
$$

where $S_{1}(\xi, \eta)$ is the set of points at distance at most 1 from the segment from $\xi$ to $\eta$. In the right hand side we can estimate $\left(d\left(\eta, N^{k+1}\right)+d\left(\zeta, N^{k+1}\right)\right)^{2}$ by a constant times $d\left(\eta, N^{k+1}\right) d\left(\xi, N^{k+1}\right)$, in view of (4.17) and (4.18). Furthermore, $1+|\eta|+|\zeta|$ can be estimated by a constant multiple of $1+|\xi|+|\eta|$. Finally, if we square and add the inequalities (4.21) for all $\beta$ and $\gamma$ with $|\beta|=(|\alpha|+1) k ;|\gamma|=(m+1) k$, and then use (4.10) to estimate the left hand side of the resulting inequality from below, we get

$$
\left|R_{m}^{(\alpha)}(\xi, \eta)\right| \leqslant K_{\mathbf{5}}|\xi-\eta|^{m-|\alpha|}(1+|\xi|+|\eta|)^{n_{s}}\left|F^{\prime}\right|_{m^{\prime}, S_{1}(\xi, \eta)}, \quad \xi, \eta \in N^{k} .
$$

Let $m^{\prime}$ be an integer such that $(4.11)^{\prime \prime}$ and (4.20) are fulfilled. Then all the estimates (4.12), (4.15), (4.22) hold when $|\alpha| \leqslant m$ (possibly for different $n^{\prime}$ and $K$ ). Hence ine- 
quality (4.3) is valid with some $n^{\prime}$ and $K$ for all $f$ vanishing of order $m^{\prime}$ in $N^{k+1}$, for $|f|_{m, N_{\xi}} k_{\xi}$ is the least upper bound of the quantities

$$
\left|f^{(\alpha)}(\eta)\right|, \quad\left|R_{m}^{(\alpha)}(\xi ; \eta)\right| /|\xi-\eta|^{m-|\alpha|}, \quad|\alpha| \leqslant m
$$

when $\xi, \eta \in N^{k}$ and $\left|\xi-\xi^{\prime}\right| \leqslant R, \quad\left|\eta-\xi^{\prime}\right| \leqslant R$.

It remains to show that (4.3) is valid without the restriction that $f$ shall vanish of order $m^{\prime}$ in $N^{k+1}$. To do so we apply Theorem 2 with $A=N^{k+1}$ and $m$ replaced by $m^{\prime}$. From (3.4) we then get a function $g \in C^{m^{\prime}}\left(R^{v}\right)$ such that, with $h=f-g$,

$$
h \in C^{m^{\prime}}\left(R^{v}\right), h=0 \text { of order } m^{\prime} \text { in } N^{k+1} \text {. }
$$

We have $f=h+g$ and from (3.12) we get

$$
|g|_{m^{\prime}, \xi} \leqslant K_{1}|f|_{m^{\prime}, N^{k+1}} .
$$

Hence, recalling that $P(\xi)$ is of degree $\mu$, we get

$$
\begin{aligned}
\sup (1+|\xi|)^{n^{\prime}}|P g|_{m^{\prime}, \xi} & \leqslant K_{2} \sup (1+|\xi|)^{n^{*}+\mu}|g|_{m^{\prime}, \xi} \\
& \leqslant K_{3} \sup (1+|\xi|)^{n^{\prime}+\mu}|f|_{m^{\prime}, N^{k+1}} .
\end{aligned}
$$

Since we have assumed that (4.3) is true when $k$ is replaced by $k+1$, we get with some $n^{\prime \prime}$ and $m^{\prime \prime}$

$$
\sup (1+|\xi|)^{n^{*}}|P g|_{m^{\prime}, \xi} \leqslant K_{4} \sup (1+|\xi|)^{n^{\prime \prime}}|P f|_{m^{\prime \prime}, \xi} .
$$

Since $h=f-g$ and $n^{\prime \prime} \geqslant n^{\prime} ; m^{\prime \prime} \geqslant m^{\prime}$, we get

$$
\sup (1+|\xi|)^{n^{\prime}}|P h|_{m^{\prime}, \xi} \leqslant\left(K_{4}+1\right) \sup (1+|\xi|)^{n^{\prime \prime}}|P f|_{m^{\prime \prime}, \xi} .
$$

Now $h$ vanishes of order $m^{\prime}$ in $N^{k+1}$ so we can apply (4.3) to $h$. This gives

$$
\sup (1+|\xi|)^{n}|h|_{m, N^{k}} \leqslant K \sup (1+|\xi|)^{n^{\prime}}|P h|_{m^{\prime}, \xi} .
$$

Combining (4.25) with (4.24) and estimating $g$ by means of (4.23) and the induction hypothesis again, we get with some $n^{\circ}, m^{\circ}$ and $K^{\circ}$

$$
\sup (1+|\xi|)^{n}|f|_{m, N^{k}} \leqslant K^{\circ} \sup (1+|\xi|)^{n^{\circ}}|P f|_{m^{\circ}, \xi}
$$

This completes the proof of (4.3) and thus of Theorem 1.

\section{Applications of Theorem 1}

We shall now show that the division problem can easily be studied by means of Theorem 1.

Theorem 3. Let $T$ be a tempered distribution and $P$ a polynomial 末 0 . Then there is a tempered distribution $S$ such that $T=P S$. 
L. HöRMANDER, On the division of distributions by polynomials

Theorem 4. If $T$ is a distribution in an open set $\Omega$, there is a distribution $S$ in $\Omega$ such that $T=P S$, where $P$ is a given polynomial $\neq 0$.

Proof of Theorem 3. Consider the linear form

$$
P f \rightarrow T(f)
$$

which is defined in a subset $P S$ of $S$. It is continuous for if $P f \rightarrow 0$ in $S$ it follows that $f \rightarrow 0$ in $S$ (Theorem 1), hence $T(f) \rightarrow 0$. In view of the Hahn-Banach theorem we can extend the linear form to a linear form $\varphi \rightarrow S(\varphi)$ on the whole of $S$. Then we have $S(P f)=T(f)$, that is, $P S=T$.

The proof of Theorem 4 is exactly analogous and can be omitted.

Theorem 5. If $P(D)$ is a partial differential operator with constant coefficients and $T$ a tempered distribution, there is a tempered distribution $S$ such that

$$
P(D) S=T .
$$

Proof. Applying the Fourier transformation in the sense of Schwartz [4], we transform (5.1) into the equation

$$
P(-i \xi) \hat{B}=\hat{T},
$$

where $S$ and $\hat{T}$ are the Fourier transforms of $S$ and $T$. Now this equation has a tempered solution $\widehat{S}$, hence inverting the Fourier transformation we obtain a solution of (5.1). The proof is complete.

REFERENCES

1. L. HöRMANDER, Local and global properties of fundamental solutions. Math. Scand. 5, 27-39 (1957).

2. S. LoJasirwicz, Division d'une distribution par une fonction analytique de variables réelles. C. R. Acad. Sci. Paris 246, 683-686 (1958) (Séance du 3 Février 1958).

3. B. Malgrange, Existence et approximation des solutions des équations aux dérivées partielles et des équations de convolution. Ann. Inst. Fourier Grenoble 6, 271-354 (1955-56).

4. L. Schwartz, Théorie des distributions, I-II. Paris, 1950-51.

5. A. Seidenbera, A new decision method for elementary algebra. Ann. of Math., (2) 60, 365-374 (1954).

6. H. Whitney, Analytic extensions of differentiable functions defined in closed sets. Trans. Amer. Math. Soc. 36, 63-89 (1934). 